\title{
Counter-Examples to the Generalized Positive Action Conjecture
}

\author{
Claude LeBrun ${ }^{\star}$
}

Department of Mathematics, State University of New York, Story Brook, New York 11794-3651, USA

\begin{abstract}
We give examples of complete locally asymptotically flat Riemannian 4-manifolds with zero scalar curvature and negative mass. The generalized positive action conjecture of Hawking and Pope [5] is therefore false.
\end{abstract}

The positive action theorem, proved by Schoen and Yau [9], states that any complete asymptotically flat Riemannian 4-manifold $(M, g)$ with scalar curvature $R=0$ satisfies

$$
\lim _{r \rightarrow \infty} \int_{S_{r}}\left(g_{j k, k}-g_{k k, j}\right) * d S^{j} \geqq 0
$$

with equality iff $g$ is flat; here the integrand is to be computed in an asymptotic coordinate system in which the metric is of the form

$$
g_{j k}=\delta_{j k}+0\left(\frac{1}{r^{2}}\right)
$$

and $S_{r}$ denotes the Euclidean sphere of radius $r$. For simplicity we will call the left-hand side of the above inequality the mass of $(M, g)$, since this expression is the analog of the ADM mass of an asymptotically flat 3-manifold. The generalized positive action conjecture of Hawking and Pope [5] asserts that the mass is also non-negative for all locally asymptotically flat Riemannian 4-manifolds with scalar curvature $R=0$, and that, moreover, the mass vanishes iff the manifold is Ricci flat with self-dual Weyl curvature.

Unfortunately, as we will see, this plausible-sounding extension does not hold water. We will produce an infinite number of complete locally asymptotically flat Riemannian 4-manifolds with $R=0$ for which the mass is negative. These metrics are Kähler and live on the total spaces of complex line-bundles over $S^{2}=\mathbb{C P}{ }_{1}$ for which the first Chern class satisfies $c_{1}<-2$. They have isometry group $U(2)$, and, by virtue of being Kähler with $R=0$, have anti-self-dual Weyl curvature; cf. [2, 7, 8].

\footnotetext{
* Research partially supported by NSF grant \#DMS-8704401
} 
It was the intrinsic interest of such anti-self-dual metrics which motivated the construction of these examples.

We begin by considering a Kähler potential $\phi(u)$, where $u=\left|z_{1}\right|^{2}+\left|z_{2}\right|^{2}$; thus

$$
\omega=i \bar{\partial} \partial \phi
$$

is the Kähler form of a metric on a spherical shell centered at the origin in $\mathbb{C}^{2}$. Letting $V(u)$ be defined by

$$
\omega \wedge \omega=-V d z_{1} \wedge d \bar{z}_{1} \wedge d z_{2} \wedge d \bar{z}_{2}
$$

the Ricci form of $\omega$ is given by

$$
\rho=i \bar{\partial} \partial \log V
$$

and the scalar curvature $R$ satisfies

$$
R \omega \wedge \omega=\rho \wedge \omega .
$$

The equation $R=0$ may therefore be written as

$$
(\partial \bar{\partial} \phi) \wedge(\partial \bar{\partial} \log V)=0 .
$$

The symmetry of the situation allows us to merely carry out our calculations at the $z_{1}$-axis. Letting $\psi=\log V$,

$$
\begin{aligned}
& \partial \bar{\partial} \phi=\left(\phi^{\prime}+u \phi^{\prime \prime}\right) d z_{1} \wedge d \bar{z}_{1}+\phi^{\prime} d z_{2} \wedge d \bar{z}_{2} \\
& \partial \bar{\partial} \psi=\left(\psi^{\prime}+u \psi^{\prime \prime}\right) d z_{1} \wedge d \bar{z}_{1}+\psi^{\prime} d z_{2} \wedge d \bar{z}_{2}
\end{aligned}
$$

at the $z_{1}$-axis, so that our equation reads

$$
0=\left(\phi^{\prime}+u \phi^{\prime \prime}\right) \psi^{\prime}+\left(\psi^{\prime}+u \psi^{\prime \prime}\right) \phi^{\prime}
$$

or

$$
0=\frac{1}{u} \frac{d}{d u}\left(u^{2} \phi^{\prime} \psi^{\prime}\right)
$$

Hence, for some constant $A, u^{2} \phi^{\prime} \psi^{\prime}=A$.

Now let $y=u \phi^{\prime}$. Then

$$
\frac{1}{2} V=\phi^{\prime}\left(\phi^{\prime}+u \phi^{\prime \prime}\right)=\frac{y y^{\prime}}{u}
$$

and

Thus

$$
\psi^{\prime}=\frac{d}{d u} \log V=\frac{y^{\prime}}{y}+\frac{y^{\prime \prime}}{y^{\prime}}-\frac{1}{u} .
$$

and

$$
A=u^{2} \phi^{\prime} \psi^{\prime}=u y^{\prime}+\frac{u y y^{\prime \prime}}{y^{\prime}}-y,
$$

$$
A y^{\prime}=u\left(y^{\prime}\right)^{2}-y y^{\prime}+u y y^{\prime \prime}=\frac{d}{d u}\left[u y y^{\prime}-y^{2}\right] .
$$


It follows that

$$
y^{2}+A y+B=u y y^{\prime} \quad \text { and } \quad \log u=\int \frac{y d y}{y^{2}+A y+B} .
$$

Hence, letting $\left(y^{2}+A y+B\right)=\left(y+\alpha_{1}\right)\left(y+\alpha_{2}\right)$,

Now

$$
u= \begin{cases}C\left(y+\alpha_{2}\right)^{\alpha_{2} /\left(\alpha_{2}-\alpha_{1}\right)}\left(y+\alpha_{1}\right)^{\alpha_{1} /\left(\alpha_{1}-\alpha_{2}\right)}, & \alpha_{1} \neq \alpha_{2} \\ C(y+\alpha) e^{\alpha /(y+\alpha)}, & \alpha_{1}=\alpha_{2}=\alpha .\end{cases}
$$

$$
\begin{aligned}
\phi= & \int \frac{y}{u} d u=y \log u-\int \log u d y=\left(y+\alpha_{1}\right) \frac{\alpha_{1}}{\alpha_{1}-\alpha_{2}}+\left(y+\alpha_{2}\right) \frac{\alpha_{2}}{\alpha_{2}-\alpha_{1}} \\
& -\frac{\alpha_{1}^{2}}{\alpha_{1}-\alpha_{2}} \log \left(y+\alpha_{1}\right)-\frac{\alpha_{2}^{2}}{\alpha_{2}-\alpha_{1}} \log \left(y+\alpha_{2}\right)+\text { const, }
\end{aligned}
$$

so

$$
\phi(u)=y-\alpha_{1} \log u-\alpha_{2} \log \left(y+\alpha_{2}\right)+\text { const, }
$$

where $y(u)$ is obtained by inverting Eq. (1).

In order to give the metric explicitly, we now introduce a new coordinate $r=\sqrt{y}$, and let $\sigma_{1}, \sigma_{2}, \sigma_{3}$ be a left-invariant coframe for $S^{3}$ coinciding with $d x^{2}$, $d y^{2}, d y^{1}$ at $\left(z_{1}, z_{2}\right)=(1,0)$. The Kähler form is then given by

$$
\frac{i \omega}{2}=\left(\phi^{\prime}+u \phi^{\prime \prime}\right)\left(d(\sqrt{u}) \wedge \sqrt{u} \sigma_{3}\right)+u \phi^{\prime}\left(\sigma_{1} \wedge \sigma_{2}\right),
$$

so that

$$
g=\frac{d y}{d u}\left(\frac{d u^{2}}{4 u}+u \sigma_{3}^{2}\right)+r^{2}\left(\sigma_{1}^{2}+\sigma_{2}^{2}\right) .
$$

But since $u y y^{\prime}=y^{2}+A y+B$,

$$
\frac{d y}{d u} \frac{d u^{2}}{4 u}=\frac{y d r^{2}}{u y^{\prime}}=\frac{y^{2} d r^{2}}{y^{2}+A y+B}=\frac{d r^{2}}{1+\frac{A}{r^{2}}+\frac{B}{r^{4}}} .
$$

Similarly,

$$
u y^{\prime} \sigma_{3}^{2}=\left(1+\frac{A}{r^{2}}+\frac{B}{r^{4}}\right) r^{2} \sigma_{3}{ }^{2} \text {, }
$$

and hence

$$
g=\frac{d r^{2}}{1+\frac{A}{r^{2}}+\frac{B}{r^{4}}}+r^{2}\left(\sigma_{1}^{2}+\sigma_{2}^{2}+\left(1+\frac{A}{r^{2}}+\frac{B}{r^{4}}\right) \sigma_{3}{ }^{2}\right)
$$

If $A=0$, this is the metric of Eguchi-Hanson [3]; it is Ricci-flat, and the potential $\phi$ is explicitly given by

$$
\phi(u)=\sqrt{u^{2}+b^{2}}+\frac{b}{2} \log \left(\sqrt{u^{2}+b^{2}}-b\right)-\frac{b}{2} \log \left(\sqrt{u^{2}+b^{2}}+b\right)
$$


for $B=-b^{2}$. (Eguchi and Hanson adopt the opposite orientation convention, and so consider the metric to be self-dual instead of anti-self-dual.)

If $B=0$, the potential $\phi$ may again be given explicitly, namely by

$$
\phi(u)=u-A \log u .
$$

Provided that $A<0$, this metric is the standard metric [4] on the blow-up of $\mathbb{C}^{2}$ at the origin; the fact that it has zero scalar curvature was discovered by Burns [1]. Its anti-self-duality is of importance because it is conformally isometric to the Fubini-study metric (in an orientation reversing manner), the map being

$$
\Phi: \mathbb{C}^{2}-\{0\} \rightarrow \mathbb{C}^{2}-\{0\}, \quad \Phi\left(z_{1}, z_{2}\right)=\left(\frac{z_{1}}{\left|z_{1}\right|^{2}+\left|z_{2}\right|^{2}}, \frac{z_{2}}{\left|z_{1}\right|^{2}+\left|z_{2}\right|^{2}}\right) .
$$

(We have restricted the Fubini-study metric to $\mathbb{C}^{2} \subset \mathbb{C} \mathbb{P}_{2}$; by dilation we take its Kähler potential to be $\log (1-A u)$, thereby making $\Phi$ the required conformal map.)

Both the Eguchi-Hanson and Burns metrics can be made complete by replacing the origin with $\mathbb{C} \mathbb{P}_{1}$ and, in the Eguchi--Hanson case, modding out by $Z_{2}$; thus, the metrics naturally live on complex line bundles over $\mathbb{C P}_{1}$. We now generalize the procedure.

Suppose that $\alpha_{1}=-a^{2}, \alpha_{2}=k a^{2}$, where $a>0$ and $k$ is a non-negative integer. Restrict attention, for a moment, to fibers of the Hopf map $\mathbb{C}^{2}-\{0\} \rightarrow \mathbb{P}_{1}$. Then $g$ becomes

$$
\left.g\right|_{\text {fiber }}=\frac{d r^{2}}{\left(1-\frac{a^{2}}{r^{2}}\right)\left(1+\frac{k a^{2}}{r^{2}}\right)}+\left(1-\frac{a^{2}}{r^{2}}\right)\left(1+\frac{k a^{2}}{r^{2}}\right) r^{2} d \theta^{2}
$$

Now divide out by the action of $Z_{k+1}$, introducing a new angular coordinate $\hat{\theta}=(k+1) \theta$, and simultaneously introducing a new radial coordinate $\hat{r}=r^{2}-a^{2}$. The fiber-wise metric thus becomes

$$
\begin{aligned}
\left.g\right|_{\text {fiber }} & =\frac{\hat{r}^{2} d \hat{r}^{2}}{\hat{r}^{2}\left(1+\frac{k a^{2}}{\hat{r}^{2}+a^{2}}\right)}+\left(1+\frac{k a^{2}}{\hat{r}^{2}+a^{2}}\right) \frac{\hat{r}^{2}}{(1+k)^{2}} d \hat{\theta}^{2} \\
& =\frac{1}{1+\frac{k a^{2}}{\hat{r}^{2}+a^{2}}}\left[d r^{2}+\left(\frac{1+\frac{k}{1+(\hat{r} / a)^{2}}}{1+k}\right)^{2}\right] .
\end{aligned}
$$

Since $\left(1+k / 1+(\hat{r} / a)^{2}\right) /(1+k)$ differs from 1 by terms of order $\hat{r}^{2}$, this metric extends smoothly across the origin $\hat{r}=0$.

Let us now notice that this process is compatible with the complex structure by inspecting Eq. 1, which in the present circumstances yields

$$
\left|z_{1}\right|^{2}+\left|z_{2}\right|^{2}=\left(r^{2}+k a^{2}\right)^{k /(k+1)}\left(r^{2}-a^{2}\right)^{1 / k+1} .
$$

If we identify the complex $\zeta$-plane with the quotient of a fiber by $Z_{k+1}$ via the map 


$$
\zeta \rightarrow\left(\frac{c_{1} \zeta^{1 /(k+1)}}{{\sqrt{c_{1}}}^{2}+c_{2}^{2}}, \frac{c_{2} \zeta^{1 /(k+1)}}{\sqrt{c_{1}^{2}+c_{2}^{2}}}\right)
$$

we obtain

$$
|\zeta|^{2}=\left(r^{2}-a^{2}\right)\left(r^{2}+k a^{2}\right)^{k}
$$

so that the radial coordinate $\hat{r}$ is smoothly related to $|\zeta|$ by

$$
|\zeta|=\hat{r}\left(\hat{r}^{2}+(k+1) a^{2}\right)^{1 / k}
$$

Essentially we same argument shows that the only other complete metric that can be obtained from the above family is the flat metric $A=B=0$. Thus, we have the following:

Proposition. Let $L \rightarrow \mathbb{C} \mathbb{P}_{1}$ denote the line bundle with Chern class +1 . For any integer $k \geqq 0, L^{-(k+1)}$ admits a complete zero scalar curvature asymptotically flat Kähler metric. Up to multiplication by an overall constant, there is exactly one such metric which is $S U(2)$ invariant.

(The last observation follow from the fact that the metric (3) is only multiplied by an overall constant if $A$ and $B$ are changed in a fashion preserving $A^{2} / B$.)

Let us note that each of these Riemannian manifolds deform retracts to $S^{2}$. They are therefore topologically much simpler than the familiar gravitational instanton solutions (cf. [6]) for which the second Betti number increases as the order of the fundamental group of infinity.

We now compute the masses of these manifolds. The coordinate system of (3) gives rise to an asymptotic coordinate system via polar coordinates on Euclidean 4-space. Thus, (3) becomes

$$
g_{j k}=\delta_{j k}-\frac{A}{r^{4}} x^{j} x^{k}+\frac{A}{r^{4}} J_{l}^{l} J_{m}^{k} x^{l} x^{m}+0\left(\frac{1}{r^{4}}\right) .
$$

where

$$
J=\left[\begin{array}{rrrr}
0 & -1 & 0 & 0 \\
1 & 0 & 0 & 0 \\
0 & 0 & 0 & -1 \\
0 & 0 & 1 & 0
\end{array}\right] .
$$

Thus

$$
\left(g_{j k . j}-g_{j j, k}\right) \frac{x^{k}}{r}=-\frac{2 A}{r^{3}}+0\left(\frac{1}{r^{5}}\right),
$$

and the mass is therefore

$$
\lim _{r \rightarrow \infty}-\frac{2 A}{r^{3}} 2 \pi^{2} r^{3}=-4 \pi^{2} A .
$$

For our complete metrics we have $A=\alpha_{1}+\alpha_{2}=(k-1) a^{2}$, so the mass is negative unless $k=0$ (the strictly asymptotically flat case) or $k=1$ (the Eguchi-Hanson case considered by the framers of the conjecture).

To sum up, we've shown the following: 
Theorem. The total space of any complex line bundle over $\mathbb{C P}_{1}$ with Chern class $c_{1}<-2$ carries a complete zero scalar curvature Kähler metric of negative mass. The generalized positive action conjecture is therefore false.

Acknowledgement. It is a pleasure to thank Mike Anderson for several helpful suggestions.

\section{References}

1. Burns, D.: Twistors and harmonic maps. Talk in Charlotte, N.C., October 1986

2. Derdzinski, A.: Compos. Math. 49, 405-433 (1983)

3. Eguchi, T., Hanson, A.J.: Ann. Phys. 120, 82-106 (1979)

4. Griffiths, P., Harris, J.: Principles of algebraic geometry. New York: Wiley Interscience

5. Hawking, C. W., Pope, C. N.: Nucl. Phys. B146, 381-392 (1978)

6. Hitchin, N. J.: Math. Proc. Camb. Phil. Soc. 85, 465-476 (1979)

7. Itoh, M.: Compos. Math. 51, 265-273 (1984)

8. LeBrun, C.: Proc. Am. Math. Soc. 98, 637-640 (1986)

9. Schoen, R., Yau. S.-T.: Phys. Rev. Lett. 42, 547-548 (1979)

Communicated by S.-T. Yau

Received December 8, 1987 